\title{
Stabilization and enhancement of traffic flow by the next-nearest-neighbor interaction
}

\author{
Takashi Nagatani \\ Division of Thermal Science, Department of Mechanical Engineering, Shizuoka University, Hamamatsu 432-8561, Japan
}

(Received 13 July 1999)

\begin{abstract}
The car-following model of traffic is extended to take into account the car interaction before the next car ahead (the next-nearest-neighbor interaction). The traffic behavior of the extended car-following model is investigated numerically and analytically. It is shown that the next-nearest-neighbor interaction stabilizes the traffic flow. The jamming transition between the freely moving and jammed phases occurs at a higher density than the threshold of the original car-following model. By increasing the maximal velocity, the traffic current is enhanced without jam by the stabilization effect. The jamming transition is analyzed with the use of the linear stability and nonlinear perturbation methods. The traffic jam is described by the kink solution of the modified Korteweg-de Vries equation. The theoretical coexisting curve is in good agreement with the simulation result. [S1063-651X(99)01512-3]
\end{abstract}

PACS number(s): 05.70.Fh, 05.70.Jk, 89.40.+k

\section{INTRODUCTION}

Recently, traffic problems have attracted considerable attention [1-3], due to the fact that traffic behavior is important in our lives. When car density increases, traffic jams appear. Traffic jams have been studied by several traffic models: car-following models [4-9], cellular automaton models [10-15], gas kinetic models [16-19], and hydrodynamic models [20-22]. The jamming transitions between freely moving traffic and jammed traffic are very similar to the conventional phase transitions and critical phenomena: the freely moving traffic and jammed traffic correspond to the gas and liquid phases, respectively [23,24]. Furthermore, it has been shown that the metastability occurs near the transition point and induces the hysteresis phenomenon $[25,26]$.

In the car-following models, the car velocity at time $t$ is determined, through the optimal velocity function, by the headway (the difference between the car position and the position of the next car ahead) at time $t-\tau$ with delay time $\tau$. The car interacts only with the next car ahead: the car affects the other car through the so-called nearest-neighbor interaction. Since the traffic flow is unidirectional the car is affected by the next car ahead and does not interact with the car behind. In the cellular automaton models, the car position and velocity are also determined by the headway. Similarly, the car interacts with the other car through the nearestneighbor interaction.

For public demand, it will be necessary to enhance the traffic current and prevent the traffic jam. In the intelligent transportation system (ITS), several traffic control systems are considered by accepting the information of the other cars. We are interested in the enhancement and stabilization of traffic flow with the help of more information about the other car positions. In particular, the information of the car position before the next car ahead may have an important effect on the traffic flow. By the help of the information of the car position before the next car ahead, it may be possible to prevent the traffic jam and enhance the traffic current. The car-following models taking into account the next but one car ahead have been unknown until now. There is an open question as to whether or not the next-nearest-neighbor in- teraction affects effectively the traffic flow. If one can stabilize the traffic flow and enhance the traffic current, the traffic system taking into account the next-nearest-neighbor interaction will be advantageous to us.

In this paper, we present the extended version of the carfollowing models to take into account the next-nearestneighbor interaction. We investigate the effect of the nextnearest-neighbor interaction on the traffic current and the jamming transition by the use of the numerical and analytical methods. We would like to address whether or not the nextnearest-neighbor interaction enhances the traffic current and stabilizes the traffic flow. We calculate the fundamental diagram (the current-density relation) and the phase diagram (the coexisting curve, the spinodal line, and the critical point) numerically. We apply the linear stability method and the nonlinear analysis to the extended car-following model. We find the kink density wave solution for the traffic jam by deriving the modified $\mathrm{KdV}$ equation from the extended model. We compare the analytical result with the simulation result.

\section{MODEL}

We present the extended version of the car-following models. We extend the car-following model to take into account the next-nearest-neighbor interaction. Newell [4] and Whitham [5] have analyzed the car-following model described by the following equation of motion of car $j$ :

$$
\frac{d x_{j}(t+\tau)}{d t}=V\left(\Delta x_{j}(t)\right),
$$

where $x_{j}(t)$ is the position of car $j$ at time $t, \Delta x_{j}(t)$ $=x_{j+1}(t)-x_{j}(t)$ is the headway of car $j$ at time $t$, and $\tau$ is the delay time.

The idea is that a driver adjusts the car velocity $d x_{j}(t) / d t$ according to the observed headway $\Delta x_{j}(t)$. The delay time $\tau$ allows for the time lag that it takes the car velocity to reach the optimal velocity $V\left(\Delta x_{j}(t)\right)$ when the traffic flow is varying. By Taylor-expanding Eq. (1), one obtains the differential equation model [6] 


$$
\frac{d^{2} x_{j}(t)}{d t^{2}}=a\left(V\left(\Delta x_{j}(t)\right)-\frac{d x_{j}(t)}{d t}\right),
$$

where $a$ is the sensitivity of a driver [6] and is given by the inverse of the delay time. Furthermore, by transforming the time derivative to the difference in Eq. (1), one can obtain the difference equation model [9]:

$$
x_{j}(t+2 \tau)=x_{j}(t+\tau)+\tau V\left(\Delta x_{j}(t)\right) .
$$

The difference equation model is more suitable for computation since the time and space variables are discrete. These models exhibit similar traffic behavior: when the car density increases, the jamming transition occurs. In these models, the motion of a car is determined by the position of the next car ahead. These models include only the nearest-neighbor interaction.

We extend the difference equation model described by Eq. (3) to take into account the next-nearest-neighbor interaction. We assume that a driver can obtain the information of the car position before the next car ahead: the driver of car $j$ can know the positions of cars $j+1$ and $j+2$. If the headway $\Delta x_{j+1}$ of the next car $j+1$ ahead is larger than $\Delta x_{j}$ of car $j$, a driver of car $j$ may wish to proceed with larger velocity than the optimal velocity $V\left(\Delta x_{j}\right)$. When the headway $\Delta x_{j+1}$ of the next car $j+1$ ahead is less than $\Delta x_{j}$ of car $j$, a driver of car $j$ may wish to proceed with less velocity than the optimal velocity $V\left(\Delta x_{j}\right)$ determined by $\Delta x_{j}$. We assume that when the headway of the next car $j+1$ ahead is larger than that of the car $j$, car $j$ moves with the larger desired velocity $V\left(\Delta x_{j}(t)\right)+\gamma\left\{V\left(\Delta x_{j+1}(t)\right)-V\left(\Delta x_{j}(t)\right)\right\}$. Here parameter $\gamma$ represents the strength of the next-nearestneighbor interaction and $0 \leqslant \gamma \leqslant 1$. Then the extended carfollowing model is described by the following equation of motion of car $j$ :

$$
\begin{aligned}
x_{j}(t+2 \tau)= & x_{j}(t+\tau)+\tau\left[V\left(\Delta x_{j}(t)\right)\right. \\
& \left.+\gamma\left\{V\left(\Delta x_{j+1}(t)\right)-V\left(\Delta x_{j}(t)\right)\right\}\right],
\end{aligned}
$$

where $\Delta x_{j+1}(t)=x_{j+2}(t)-x_{j+1}(t)$ is the headway of the next car $j+1$ ahead at time $t . x_{j+2}$ denotes the position of the next car $j+2$ but one car ahead. When $\gamma=0$, Eq. (4) of the extended car-following model reduces to Eq. (3) of the car-following model. It is convenient to rewrite Eq. (4) in terms of the headway. One obtains the following equation:

$$
\begin{aligned}
& \Delta x_{j}(t+2 \tau)-\Delta x_{j}(t+\tau)-\tau\left[V\left(\Delta x_{j+1}(t)\right)-V\left(\Delta x_{j}(t)\right)\right] \\
& \quad-\tau \gamma\left[V\left(\Delta x_{j+2}(t)\right)-2 V\left(\Delta x_{j+1}(t)\right)+V\left(\Delta x_{j}(t)\right)\right]=0 .
\end{aligned}
$$

The last term on the left side of Eq. (5) represents the additional term of the next-nearest-neighbor interaction.

Generally, it is necessary that the optimal velocity function has the following properties: It is a monotonically increasing function and it has an upper bound (maximal velocity). We choose the same optimal velocity function as that used by Bando et al. [6],

$$
V\left(\Delta x_{j}\right)=\frac{\nu_{\max }}{2}\left\{\tanh \left(\Delta x_{j}-h_{c}\right)+\tanh \left(h_{c}\right)\right\},
$$

where $h_{c}$ is the safety distance.

When $\Delta x_{j} \rightarrow \infty$, the optimal velocity reaches the maximal velocity $V(\infty)=\nu_{\max } / 2\left\{1+\tanh \left(h_{c}\right)\right\}$. Furthermore, when $h_{c}$ $\gg 0, V(\infty) \cong \nu_{\max }$ : the maximal value of the optimal velocity is $\nu_{\max }$ for $\Delta x_{j} \gg h_{c} \gg 0$. Equation (6) has a turning point (inflection point) at $\Delta x_{j}=h_{c}: \quad V^{\prime \prime}\left(h_{c}\right)=d^{2} V\left(\Delta x_{j}\right) /$ $\left.d \Delta x_{j}^{2}\right|_{\Delta x_{j}=h_{c}}=0$. It is important that the optimal velocity function has the turning point. Otherwise, one cannot have the kink-antikink density wave solution representing the traffic jam.

\section{LINEAR STABILITY ANALYSIS}

We apply the linear stability method to the extended model described by Eq. (4). We consider the stability of the uniform traffic flow. The uniform traffic flow is defined by such a state that all cars move with constant headway $h$ and optimal velocity $V(h)$. The solution of the uniformly steady state in Eq. (4) is given by

$$
x_{j, 0}(t)=h j+V(h) t \quad \text { with } \quad h=L / N,
$$

where $N$ is the number of cars, $L$ is the system size, and $h$ is the car spacing (identical headway).

Let $y_{j}(t)$ be small deviations from the uniform solution $x_{j, 0}(t): \quad x_{j}(t)=x_{j, 0}(t)+y_{j}(t)$. Then the linear equation is obtained from Eq. (5),

$$
\begin{gathered}
\Delta y_{j}(t+2 \tau)-\Delta y_{j}(t+\tau)-\tau V^{\prime}(h)\left[\Delta y_{j+1}(t)-\Delta y_{j}(t)\right] \\
-\tau \gamma V^{\prime}(h)\left[\Delta y_{j+2}(t)-2 \Delta y_{j+1}(t)+\Delta y_{j}(t)\right]=0,
\end{gathered}
$$

where $V^{\prime}(h)$ is the derivative of optimal velocity $V(\Delta x)$ at $\Delta x=h$ and $\Delta y_{j}(t)=y_{j+1}(t)-y_{j}(t)$.

By expanding $\Delta y_{j}(t)=Y \exp (i k j+z t)$, one obtains

$$
e^{2 z \tau}-e^{z \tau}-\tau V^{\prime}(h)\left(e^{i k}-1\right)-\tau \gamma V^{\prime}(h)\left(e^{2 i k}-2 e^{i k}+1\right)=0 .
$$

By solving Eq. (9) with $z$, one finds that the leading term of $z$ is order of $i k$. When $i k \rightarrow \infty, z \rightarrow 0$. Let us derive the long wave expansion of $z$, which is determined order by order around $i k \approx 0$. By expanding $z=z_{1}(i k)+z_{2}(i k)^{2}+\cdots$, the first- and second-order terms of $i k$ are obtained,

$$
z_{1}=V^{\prime}(h) \quad \text { and } \quad z_{2}=-\frac{3}{2} \tau V^{\prime}(h)^{2}+\frac{(1+2 \gamma)}{2} V^{\prime}(h) .
$$

If $z_{2}$ is a negative value, the uniformly steady-state flow becomes unstable for long-wavelength modes. When $z_{2}$ is a positive value, the uniform flow is stable. The neutral stability condition is given as

$$
\tau=\frac{(1+2 \gamma)}{3 V^{\prime}(h)}
$$

For small disturbances of long wavelengths, the uniformly traffic flow is unstable if

$$
\tau>\frac{(1+2 \gamma)}{3 V^{\prime}(h)}
$$




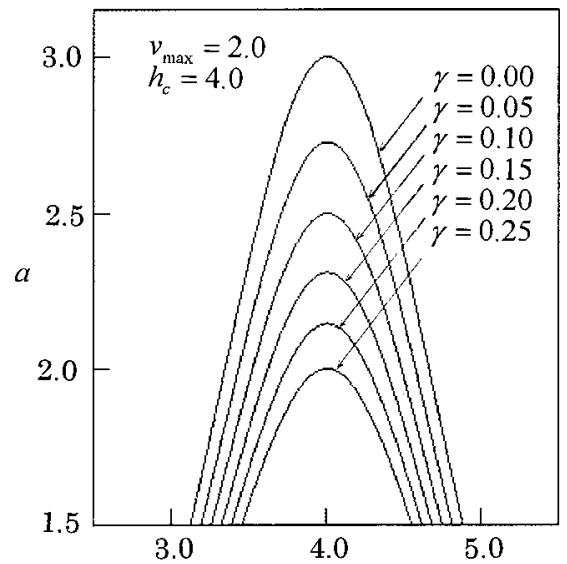

(a) $\Delta x$

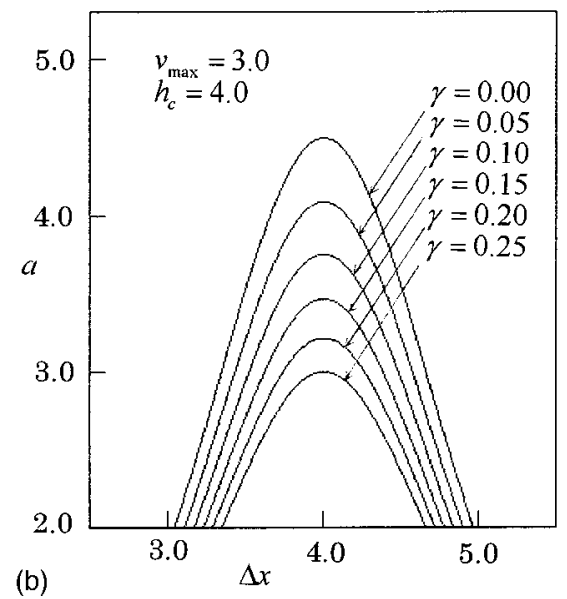

FIG. 1. Neutral stability lines in the headway $\Delta x$-sensitivity $a$ space where the safety distance $h_{c}$ is 4.0 . The solid lines indicate the neutral stability lines for various values of $\gamma$, where $\gamma$ is the strength of the next-nearest-neighbor interaction. (a) $\nu_{\max }=2.0$. (b) $\nu_{\max }=3.0$.

The derivative $V^{\prime}(h)$ of the optimal velocity has the maximal value $\nu_{\max } / 2$ at turning point $h=h_{c}$. Therefore, if $\tau$ $<\tau_{c}\left[\tau_{c}=(1+2 \gamma) / 3 \nu_{\text {max }}\right]$, the uniform flow is always stable irrespective of car density (headway). We find that there is a critical point at $h(=\Delta x)=h_{c}$ and $\tau=\tau_{c}$. When $\gamma=0$, the critical point and the neutral stability line agree with those of the original car-following model $[7,23]$. Figure 1 shows the neutral stability lines in the space $(\Delta x, a)$ for (a) $\nu_{\max }=2.0$ and (b) $\nu_{\max }=3.0$, where $\Delta x$ is the headway and $a$ is the sensitivity. The solid curves indicate the neutral stability lines for various values of $\gamma$. The neutral stability lines decrease with increasing $\gamma$. With the increase of the strength of the next-nearest-neighbor interaction, the traffic flow is more stable than that without the next-nearest-neighbor interaction. The apex of each curve indicates the critical point. The traffic flow above each curve is stable and the traffic jam does not appear. Below each curve, the traffic flow is unstable and the density waves appear. Figure 2 shows the critical line for $\nu_{\max }=2.0$. The critical line is obtained by plotting the critical point against $\gamma$. The solid line indicates the critical line. The circular points indicate the simulation result obtained in Sec. IV. The critical points decrease with increasing $\gamma$. This means that by introducing the nextnearest-neighbor interaction into the original car-following model, the traffic flow becomes more stable than that without

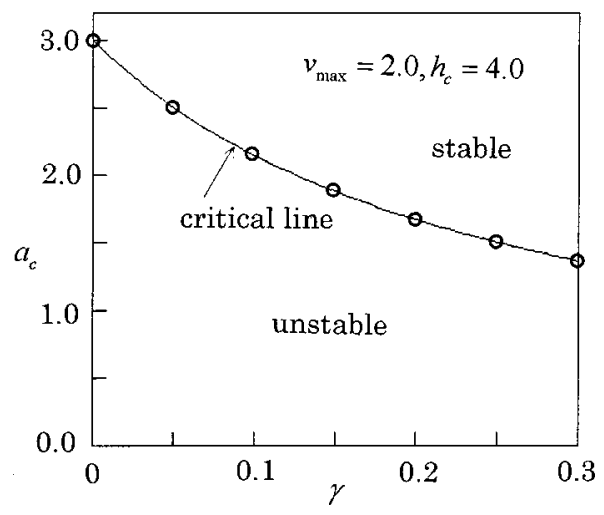

FIG. 2. Plot of the critical point against $\gamma$ for $\nu_{\max }=2.0$. The solid curve indicates the theoretical result obtained by the linear stability analysis. The circular points indicate the simulation result.

the next-nearest-neighbor interaction. The traffic current increases with $\nu_{\max }$ but the traffic flow is unstable more than that of small $\nu_{\max }$. Therefore, the simultaneous increase of the next-nearest-neighbor interaction with $\nu_{\max }$ is able to enhance the traffic current without traffic jams.

\section{SIMULATION}

We carry out a computer simulation for the extended traffic flow model described by Eq. (5). The boundary is periodic. First, we study the space-time evolution of the headway for various values of next-nearest-neighbor interaction $\gamma$. As a result, three types of traffic flow are distinguished similarly to the original optimal velocity model: (i) a freely moving phase, (ii) a coexisting phase in which jams appear, and (iii) a uniformly congested phase. We focus our attention on the coexisting phase. Figure 3 shows the steady-state patterns for the coexisting phase obtained after $t=10000$. For these patterns, the initial conditions are chosen as follows: $\Delta x_{j}(0)$ $=\Delta x_{0}=4.0$ for $j \neq 50,51, \Delta x_{j}(0)=\Delta x_{0}=4.0-0.1$ for $j$ $=50$, and $\Delta x_{j}(0)=\Delta x_{0}=4.0+0.1$ for $j=51$, where the number of cars is $N=100$ and $h_{c}=4.0$. The patterns (a), (b), and (c) exhibit the time evolutions of the headway profile for $\gamma=0.0,0.1,0.2$, where $\nu_{\max }=2.0$ and $a(=1 / \tau)=2.0$. The traffic jams propagate backward as the kink-antikink density wave. The pattern (a) with $\gamma=0.0$ corresponds to that without the next-nearest-neighbor interaction. With increasing $\gamma$, the strength of the density waves is weakened by the nextnearest-neighbor interaction. Figure 4 shows the headway profiles in the steady state (obtained at $t=20000$ ) for various values of $\gamma$ and $\nu_{\max }=2.0$. When the value of $\gamma$ is larger than the critical value, the density waves do not appear and the traffic flow is uniform over space [see Fig. 4(c)]. In any case, considering long-time evolution only two distinct headways survive for the coexisting phase: one is the headway within the jam and the other is the headway out of the jam corresponding to that in the freely moving phase. These headways depend on the delay time $\tau$ (the inverse of the sensitivity $a$ ) and the strength $\gamma$ of the next-nearest-neighbor interaction. They are the headways of the jamming transition points. By plotting the headways within and out of the density wave, one obtains the coexisting curve in the phase space $(\Delta x, a)$. Figure 5 shows the phase diagrams in the space $(\Delta x, a)$ for (a) $\nu_{\max }=2.0$ and (b) $\nu_{\max }=3.0$. In each 


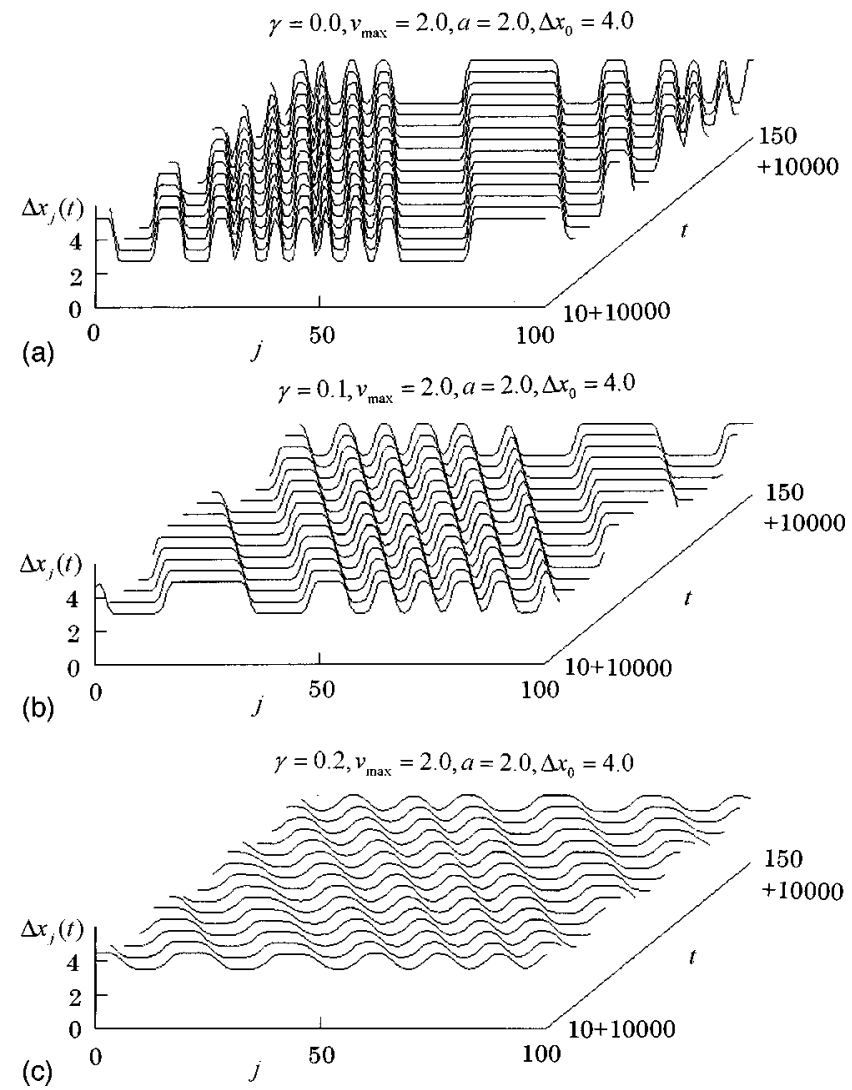

FIG. 3. Steady-state patterns for the coexisting phase obtained after $t=10000$. The patterns (a), (b), and (c) show the time evolutions of the headway profile for $\gamma=0.0,0.1,0.2$, where $\nu_{\max }=2.0$ and $a=2.0$

phase diagram, the solid line for $\gamma=0.0$ represents the coexisting curve in the original optimal velocity model without the next-nearest-neighbor interaction. The dotted line indicates the neutral stability line obtained by Sec. III. The neutral stability line corresponds to the spinodal line in the firstorder phase transition. The simulation data for $\gamma=0.1$ and 0.2 are plotted by the circular points. The solid lines for $\gamma$ $=0.1$ and 0.2 represent the theoretical curves obtained from the solution of the modified $\mathrm{KdV}$ equation. The theoretical result agrees with the simulation result. The coexisting curve decreases with increasing $\gamma$. This means that the jamming transition occurs at a smaller headway (a higher density) than that without the next-nearest-neighbor interaction. Above the apex of each curve, the jamming transition does not occur. Each apex corresponds to the critical point. The simulation result of the critical point is plotted by the circular points in Fig. 2. Thus, by introducing the next-nearest-neighbor interaction into the original car-following model, the jamming transition does not occur until the car density reaches higher density than that without the next-nearest-neighbor interaction. Therefore, the next-nearest-neighbor interaction stabilizes the traffic flow. The stabilization effect is strengthened with the increase of the next-nearest-neighbor interaction.

We calculate the traffic current. We study the effect of the next-nearest-neighbor interaction on the traffic current. Figure 6 shows the plots of the traffic current $q$ against density $\rho$ for the cases of $\nu_{\max }=3.2$ and $\gamma=0.0, \nu_{\max }=3.2$ and $\gamma$ $=0.2, \quad \nu_{\max }=1.8$ and $\gamma=0.0$, and $\nu_{\max }=1.8$ and $\gamma=0.2$,
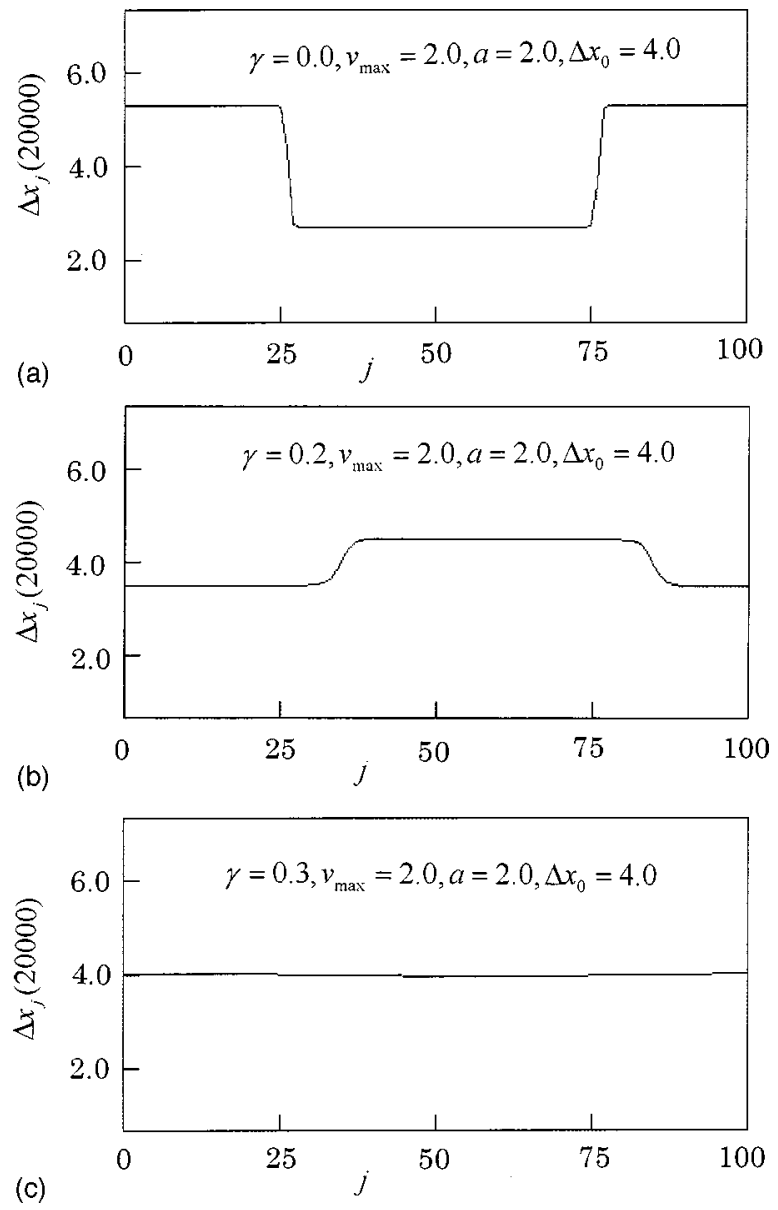

FIG. 4. Headway profiles (a), (b), and (c) in the steady state for $\gamma=0.0,0.2,0.3$, where $\nu_{\max }=2.0$ and $a=2.0$. For (a) $\gamma=0.0$ and (b) $\gamma=0.2$, the kink-antikink density wave appears. For (c) $\gamma=0.3$, the kink density wave disappears.

where $a=2.0$ and $h_{c}=4.0$. There is a gap in the traffic current. The traffic current increases with density in the lowdensity region, reaches the maximal value, decreases discontinuously at the gap, and then decreases continuously with increasing density. The gap corresponds to the jamming transition point. With increasing the maximal velocity, the traffic current increases. With increasing the strength $\gamma$ of the nextnearest-neighbor interaction, the jamming transition occurs at a higher density than that of $\gamma=0.0$. For the case of $\nu_{\max }=1.8$ and $\gamma=0.2$, no jamming transition occurs and there is no gap in the current-density relation. The traffic jam is prevented by the next-nearest-neighbor interaction. Even if the maximal velocity increases, it is possible to prevent the jamming transition. We find that it is necessary to increase the maximal velocity and the strength of the next-nearestneighbor interaction simultaneously in order to enhance the traffic current without an occurring traffic jam. Thus, the next-nearest-neighbor interaction has an important effect on the traffic flow. Taking into account the next-nearestneighbor interaction will be useful to improve the transportation system.

\section{NONLINEAR ANALYSIS}

We analyze the extended car-following model by the use of the nonlinear analysis method. We derive the modified 

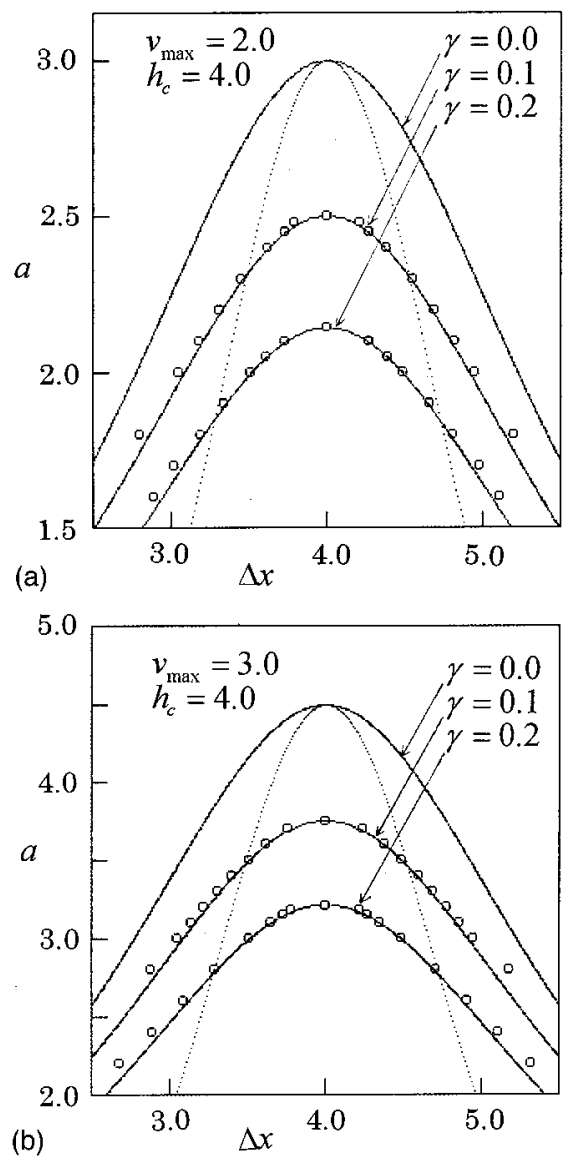

FIG. 5. Phase diagrams in the headway $\Delta x$-sensitivity $a$ space for (a) $\nu_{\max }=2.0$ and (b) $\nu_{\max }=3.0$. In each phase diagram, the solid lines represent the coexisting curves for $\gamma=0.0,0.1,0.2$. The dotted line indicates the neutral stability curve (spinodal line) for $\gamma=0.0$. The circular points indicate the simulation result.

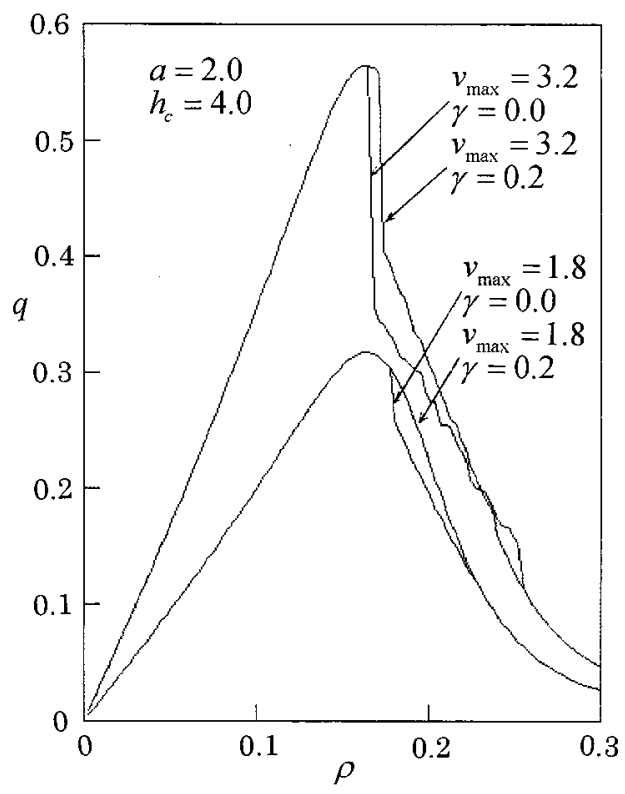

FIG. 6. Plots of the traffic current $q$ against density $\rho$ for the cases of $\mathrm{v}_{\max }=3.2$ and $\gamma=0.0, \nu_{\max }=3.2$ and $\gamma=0.2, \nu_{\max }=1.8$ and $\gamma=0.0$, and $\nu_{\max }=1.8$ and $\gamma=0.2$, where $a=2.0$ and $h_{c}=4.0$. The gap in each curve corresponds to the jamming transition point.
$\mathrm{KdV}$ equation describing the kink density wave. We find the solution of the modified $\mathrm{KdV}$ equation for the kink jam. We calculate the coexisting curve for the jamming transition from the solution. We compare the analytical result with the simulation result.

We now consider long-wavelength modes in the traffic flow on coarse-grained scales. The simplest way to describe the long-wavelength modes is the long-wave expansion. We consider the slowly varying behavior at long wavelengths near the critical point $\left(h_{c}, a_{c}\right)$. We extract slow scales for space variable $j$ and time variable $t[7,8,23,24,27]$. For 0 $<\varepsilon \ll 1$, we therefore define slow variables $X$ and $T$,

$$
X=\varepsilon(j+b t) \quad \text { and } \quad T=\varepsilon^{3} t
$$

where $b$ is a constant determined later. We set the headway as

$$
\Delta x_{j}(t)=h_{c}+\varepsilon R(X, T) .
$$

By expanding Eq. (5) to the fifth order of $\varepsilon$ with the use of Eqs. (13) and (14), one obtains the following nonlinear partial differential equation:

$$
\begin{aligned}
\varepsilon^{2}(b & \left.-V^{\prime}\right) \partial_{X} R+\varepsilon^{3}\left(\frac{3 b^{2} \tau}{2}-\frac{(1+2 \gamma)}{2} V^{\prime}\right) \partial_{X}^{2} R \\
& +\varepsilon^{4}\left[\partial_{T} R+\left(\frac{7 b^{3} \tau^{2}}{6}-\frac{V^{\prime}}{6}-\gamma V^{\prime}\right) \partial_{X}^{3} R-\frac{V^{\prime \prime \prime}}{6} \partial_{X} R^{3}\right] \\
& +\varepsilon^{5}\left[3 b \tau \partial_{X} \partial_{T} R+\left(\frac{5 b^{4} \tau^{3}}{8}-\frac{V^{\prime}}{24}-\frac{7 \gamma V^{\prime}}{12}\right) \partial_{X}^{4} R\right. \\
& \left.-\left(\frac{V^{\prime \prime \prime}}{12}+\frac{\gamma V^{\prime}}{6}\right) \partial_{X}^{2} R^{3}\right]=0
\end{aligned}
$$

where $\quad V^{\prime}=d V(\Delta x) /\left.d \Delta x\right|_{\Delta x=h_{c}} \quad$ and $\quad V^{\prime \prime \prime}$ $=d^{3} V(\Delta x) /\left.d \Delta x^{3}\right|_{\Delta x=h_{c}}$. Here we used the expansions shown in the Appendix.

By taking $b=V^{\prime}$, the second-order term of $\varepsilon$ is eliminated from Eq. (15). We consider the neighborhood of the critical point $\tau_{c}$ :

$$
\frac{\tau}{\tau_{c}}=1+\varepsilon^{2},
$$

where $\tau_{c}=(1+2 \gamma) / 3 V^{\prime}=2(1+2 \gamma) / 3 \nu_{\max }$. Equation (16) is rewritten as

$$
\begin{aligned}
& \varepsilon^{4}\left(\partial_{T} R-\frac{\left(1+13 \gamma-14 \gamma^{2}\right)}{27} V^{\prime} \partial_{X}^{3} R-\frac{V^{\prime \prime \prime}}{6} \partial_{X} R^{3}\right) \\
& \quad+\varepsilon^{5}\left(\frac{(1+2 \gamma)}{2} V^{\prime} \partial_{X}^{2} R+\frac{\left(1+6 \gamma+39 \gamma^{2}-46 \gamma^{3}\right)}{54}\right. \\
& \left.\quad \times V^{\prime} \partial_{X}^{4} R+\frac{(1+2 \gamma)}{12} V^{\prime \prime \prime} \partial_{X}^{2} R^{3}\right)=0
\end{aligned}
$$

In order to derive the regularized equation, we make the following transformation for Eq. (17): 


$$
T^{\prime}=\frac{\left(1+13 \gamma-14 \gamma^{2}\right) V^{\prime}}{27} T
$$

and

$$
R=\left(-\frac{2\left(1+13 \gamma-14 \gamma^{2}\right) V^{\prime}}{9 V^{\prime \prime \prime}}\right)^{1 / 2} R^{\prime}
$$

where

$$
1+13 \gamma-14 \gamma^{2} \leqslant 0 \text { for } 0 \leqslant \gamma \leqslant 1
$$

One obtains the regularized equation

$$
\begin{aligned}
\partial_{T^{\prime}} R^{\prime}= & \partial_{X}^{3} R^{\prime}-\partial_{X} R^{\prime 3}-\varepsilon\left(\frac{27}{2} C_{1} \partial_{X}^{2} R^{\prime}\right. \\
& \left.+\frac{1}{2} C_{2} \partial_{X}^{4} R^{\prime}-\frac{1}{2} C_{3} \partial_{X}^{2} R^{\prime 3}\right)
\end{aligned}
$$

where

$$
\begin{gathered}
C_{1}=\frac{(1+2 \gamma)}{\left(1+13 \gamma-14 \gamma^{2}\right)}, \\
C_{2}=\frac{\left(1+6 \gamma+39 \gamma^{2}-46 \gamma^{3}\right)}{\left(1+13 \gamma-14 \gamma^{2}\right)}, \quad C_{3}=1+2 \gamma .
\end{gathered}
$$

If we ignore the $O(\varepsilon)$ terms in Eq. (20), this is just the modified $\mathrm{KdV}$ equation with a kink solution as the desired solution,

$$
R_{0}^{\prime}\left(X, T^{\prime}\right)=\sqrt{c} \tanh \sqrt{c / 2}\left(X-c T^{\prime}\right)
$$

The selected value of propagation velocity $c$ for the kink solution is determined from the $O(\varepsilon)$ term.

Next, assuming that $R^{\prime}\left(X, T^{\prime}\right)=R_{0}^{\prime}\left(X, T^{\prime}\right)+\varepsilon R_{1}^{\prime}\left(X, T^{\prime}\right)$, we take into account the $O(\varepsilon)$ correction. In order to determine the selected value of the propagation velocity $c$ for the kink solution (21), it is necessary to satisfy the solvability condition

$$
\left(R_{0}^{\prime}, M\left[R_{0}^{\prime}\right]\right) \equiv \int_{-\infty}^{\infty} d X R_{0}^{\prime}\left(X, T^{\prime}\right) M\left[R_{0}^{\prime}\left(X, T^{\prime}\right)\right]=0,
$$

where

$$
M\left[R_{0}^{\prime}\right]=\frac{27}{2} C_{1} \partial_{X}^{2} R_{0}^{\prime}+\frac{1}{2} C_{2} \partial_{X}^{4} R_{0}^{\prime}-\frac{1}{2} C_{3} \partial_{X}^{2} R_{0}^{\prime 3} .
$$

By performing the integration, one obtains the selected velocity

$$
c=\frac{135 C_{1}}{2 C_{2}+3 C_{3}} \text {. }
$$

One finds the solution of the modified $\mathrm{KdV}$ equation (17),

$$
\begin{aligned}
R(X, T)= & \left(-\frac{2\left(1+13 \gamma-14 \gamma^{2}\right) V^{\prime} c}{9 V^{\prime \prime \prime}}\right)^{1 / 2} \\
& \times \tanh \sqrt{c / 2}\left(X-\frac{\left(1+13 \gamma-14 \gamma^{2}\right) V^{\prime} c}{27} T\right) .
\end{aligned}
$$

If we adopt the explicit form (6) of the optimal velocity $\left(V^{\prime}=\nu_{\max } / 2, V^{\prime \prime \prime}=-\nu_{\max }\right)$, the amplitude $A$ of the kink solution is given by

$$
A=\left[\frac{\left(1+13 \gamma-14 \gamma^{2}\right) c}{9}\left(\frac{a_{c}}{a}-1\right)\right]^{1 / 2} \quad \text { with }
$$

$$
a_{c}=\tau_{c}^{-1}=\frac{3 \nu_{\max }}{2(1+2 \gamma)} .
$$

The kink solution represents the coexisting phase, which consists of the freely moving phase with low density and the jammed (or congested) phase with high density. The headways (the inverse of the density) of the freely moving phase and the jammed phase are given, respectively, by $\Delta x=h_{c}$ $+A$ and $\Delta x=h_{c}-A$. Thus, we obtain the coexisting curve in the $(\Delta x, a)$ plane where $\Delta x$ is the headway and $a$ is the sensitivity (the inverse of delay time $\tau$ ). Figure 5 shows the coexisting curves together with the simulation result for (a) $\nu_{\max }=2.0$ and (b) $\nu_{\max }=3.0$. The solid lines indicate the coexisting curves for $\gamma=0.0,0.1$, and 0.2 . The case of $\gamma$ $=0.0$ represents the coexisting curve without the nextnearest-neighbor interaction. The circular points indicate the simulation result. The theoretical result is in good agreement with the simulation result. It is shown that the solution of the modified $\mathrm{KdV}$ equation gives the coexisting curve in the jamming transition. With increasing $\gamma$, the coexisting curve and the critical point decrease. The next-nearest-neighbor interaction stabilizes the traffic flow. The occurrence of a traffic jam is reduced to the low value of the sensitivity. Therefore, the next-nearest-neighbor interaction has an important effect on the stability of the traffic flow.

\section{SUMMARY}

We have presented the extended car-following model to take into account the next-nearest-neighbor interaction. We have investigated the effect of the next-nearest-neighbor interaction on the traffic flow by the use of numerical and analytical methods. We have shown that the next-nearestneighbor interaction stabilizes the traffic flow. We have found that the jamming transition occurs at a higher density than that without the next-nearest-neighbor interaction. We have analyzed the extended model by the use of linear stability and nonlinear methods. We have shown that the traffic jam in the traffic flow with the next-nearest-neighbor interaction is described by the modified $\mathrm{KdV}$ equation. The analytical result of the coexisting curve is in good agreement with that obtained by the simulation. We have shown that it is possible to enhance the traffic current without jam by increasing the maximal velocity and the strength of the nextnearest-neighbor interaction.

To our knowledge, this paper is the first work showing that the next-nearest-neighbor interaction has an important effect on the traffic behavior. It will be expected that the help of the information of the car position before the next car ahead results in the enhancement of traffic current and the prevention of the traffic jam. 


\section{APPENDIX}

In this appendix, we present the expansions of each term in Eq. (5) to fifth order of $\varepsilon$,

$$
\begin{aligned}
\Delta x_{j}(t+\tau)= & h_{c}+\varepsilon R+\varepsilon^{2} b \tau \partial_{X} R+\varepsilon^{3} \frac{(b \tau)^{2}}{2} \partial_{X}^{2} R \\
& +\varepsilon^{4} \frac{(b \tau)^{3}}{6} \partial_{X}^{3} R+\varepsilon^{4} \tau \partial_{T} R+\varepsilon^{5} \frac{(b \tau)^{4}}{24} \partial_{X}^{4} R \\
& +\varepsilon^{5} b \tau^{2} \partial_{T} \partial_{X} R, \\
\Delta x_{j}(t+2 \tau)= & h_{c}+\varepsilon R+\varepsilon^{2} 2 b \tau \partial_{X} R+\varepsilon^{3} \frac{(2 b \tau)^{2}}{2} \partial_{X}^{2} R \\
& +\varepsilon^{4} \frac{(2 b \tau)^{3}}{6} \partial_{X}^{3} R+\varepsilon^{4} 2 \tau \partial_{T} R+\varepsilon^{5} \frac{(2 b \tau)^{4}}{24} \partial_{X}^{4} R \\
& +\varepsilon^{5} 4 b \tau^{2} \partial_{T} \partial_{X} R,
\end{aligned}
$$

$$
\Delta x_{j+1}(t)=h_{c}+\varepsilon R+\varepsilon^{2} \partial_{X} R+\frac{\varepsilon^{3}}{2} \partial_{X}^{2} R+\frac{\varepsilon^{4}}{6} \partial_{X}^{3} R+\frac{\varepsilon^{5}}{24} \partial_{X}^{4} R,
$$

$$
\begin{aligned}
\Delta x_{j+2}(t)= & h_{c}+\varepsilon R+\varepsilon^{2} 2 \partial_{X} R+\frac{\varepsilon^{3} 4}{2} \partial_{X}^{2} R+\frac{\varepsilon^{4} 8}{6} \partial_{X}^{3} R \\
& +\frac{\varepsilon^{5} 16}{24} \partial_{X}^{4} R .
\end{aligned}
$$

We expand the optimal velocity function at the turning point:

$$
V\left(\Delta x_{j}\right)=V\left(h_{c}\right)+V^{\prime}\left(h_{c}\right)\left(\Delta x_{j}-h_{c}\right)+\frac{V^{\prime \prime \prime}\left(h_{c}\right)}{6}\left(\Delta x_{j}-h_{c}\right)^{3} .
$$

By inserting Eqs. (A1)-(A5) into Eq. (5), one obtains Eq. (15).
[1] Traffic and Granular Flow, edited by D. E. Wolf, M. Schreckenberg, and A. Bachem (World Scientific, Singapore, 1996).

[2] D. Helbing, Verkehrsdynamik (Springer, Berlin, 1997).

[3] Traffic and Granular Flow 97, edited by M. Schreckenberg and D. E. Wolf (Springer, Singapore, 1998).

[4] G. F. Newell, Oper. Res. 9, 209 (1961).

[5] G. B. Whitham, Proc. R. Soc. London, Ser. A 428, 49 (1990).

[6] M. Bando, K. Hasebe, A. Nakayama, A. Shibata, and Y. Sugiyama, Phys. Rev. E 51, 1035 (1995).

[7] T. Komatsu and S. Sasa, Phys. Rev. E 52, 5574 (1995).

[8] T. Nagatani and K. Nakanishi, Phys. Rev. E 57, 6415 (1998).

[9] T. Nagatani, K. Nakanishi, and H. Emmerich, J. Phys. A 31, 5431 (1998).

[10] K. Nagel and M. Schreckenberg, J. Phys. I 2, 2221 (1992).

[11] O. Biham, A. A. Middleton, and D. A. Levine, Phys. Rev. A 46, R6124 (1992).

[12] T. Nagatani, Phys. Rev. E 48, 3290 (1993).

[13] J. A. Cuesta, F. C. Matinez, J. M. Nolera, and A. Sanchez, Phys. Rev. E 48, 4175 (1993).

[14] K. Nagel, D. E. Wolf, P. Wagner, and P. Simon, Phys. Rev. E 58, 1425 (1998).
[15] D. Helbing and M. Schreckenberg, Phys. Rev. E 59, R2505 (1999).

[16] E. Ben-Naim, P. L. Krapivsky, and S. Redner, Phys. Rev. E 50, 822 (1994).

[17] D. Helbing, Phys. Rev. E 53, 2366 (1996).

[18] M. Treiber, A. Hennecke, and D. Helbing, Phys. Rev. E 59, 239 (1999)

[19] T. Nagatani, Physica A 237, 67 (1997).

[20] B. S. Kerner, P. Konhauser, and M. Schilke, Phys. Rev. E 51, 6243 (1995).

[21] T. Nagatani, Physica A 261, 599 (1998).

[22] H. Y. Lee, H. W. Lee, and D. Kim, Phys. Rev. E 59, 5101 (1999).

[23] T. Nagatani, Phys. Rev. E 58, 4271 (1998).

[24] T. Nagatani, Phys. Rev. E 59, 4857 (1999).

[25] S. Krauss, P. Wagner, and C. Gawron, Phys. Rev. E 55, 5597 (1997).

[26] B. S. Kerner and H. Rehborn, Phys. Rev. E 53, R1297 (1996).

[27] M. C. Cross and P. C. Hohenberg, Rev. Mod. Phys. 65, 851 (1993). 